\title{
Erneute Zunahme der registrierten Fälle von Kindsmisshandlung
}

\section{Markus Wopmann}

Dr. med., Leiter Fachgruppe Kinderschutz der Schweizerischen Kinderkliniken, Chefarzt Klinik für Kinder und Jugendliche Kantonsspital Baden

Im Jahre 2014 wurden im sechsten Jahr in Folge die Kinder erfasst, die wegen vermuteter oder sicherer Kindsmisshandlung ambulant oder stationär an einer schweizerischen Kinderklinik behandelt worden waren.

\section{In jedem 5. Fall entschloss sich die Kinder-} schutzgruppe zur Gefährdungsmeldung an die Kindes- und Erwachsenenschutzbehörde.

Erfreulicherweise konnten dieses Jahr die Daten von 21 der insgesamt 26 Kinderkliniken der Schweiz bearbeitet werden, was einem Rücklauf von $80 \%$ entspricht. Die 5 Kinderkliniken oder Kinderabteilungen, die sich nicht an der Erfassung beteiligt haben, dürften aufgrund ihrer allgemeinen Fallzahlen nur wenig Fälle von Kindsmisshandlungen betreut haben, so dass die Anzahl der uns gemeldeten Fälle einen sehr grossen Teil der Fälle von Kindsmisshandlung darstellen, die an den schweizerischen Kinderkliniken im Jahre 2014 behandelt worden waren.

\section{Resultate}

Von den 21 Kliniken wurden insgesamt 1405 Fälle gemeldet, was eine Zunahme um weitere $9 \%$ gegenüber dem Vorjahr bedeutet! Die Fallzahl stieg in den letzten sechs Jahren konstant an und erreichte einen neuen Höchstwert. Ein Drittel der Zunahme der Fälle ist auf die drei Kliniken zurückzuführen, die uns 2014 erstmals Daten zur Verfügung gestellt haben, die restliche Zunahme resultiert vor allem aus einer höheren Fallzahl an einigen grossen Kinderkliniken. Eine hohe Sensibilisierung aller Berufsgruppen an den Kinderkliniken sowie eine gute Erfassung tragen höchstwahrscheinlich massgeblich zu dieser Zunahme bei. In den verschiedenen Untergruppen ergaben sich folgende Zahlen:

\begin{tabular}{lrc}
\hline Körperliche Misshandlung & 396 & $(28,2 \%)$ \\
\hline Vernachlässigung & 307 & $(21,9 \%)$ \\
\hline Psychische Misshandlung & 379 & $(27,0 \%)$ \\
\hline Sexueller Missbrauch & 319 & $(22,7 \%)$ \\
\hline Münchhausen-Stellvertreter-Syndrom & 4 & $(0,3 \%)$ \\
\hline
\end{tabular}

Über die letzten Jahre und auch gegenüber dem letzten Jahr hat der Anteil der Kinder, die psychisch misshandelt worden waren, erneut zugenommen. Darunter fallen Kinder, die beispielsweise verbal gedemütigt, bedroht oder genötigt werden, zudem aber auch Kinder, die durch das Miterleben von häuslicher Gewalt (unter den Eltern) stark betroffen und verängstigt sind. Ein Fünftel aller misshandelten Kinder war jünger als 2 Jahre, gut 40\% der misshandelten Kinder sind jünger als 6 Jahre. Insgesamt waren - wie letztes Jahr - 45\% Knaben und 55\% Mädchen betroffen.

Geschlecht der Kinder in den einzelnen Diagnosegruppen

\begin{tabular}{lll} 
& Knaben & Mädchen \\
\hline Körperliche Misshandlung & $53,3 \%$ & $46,7 \%$ \\
\hline Vernachlässigung & $50,5 \%$ & $49,5 \%$ \\
\hline Psychische Misshandlung & $49,3 \%$ & $50,7 \%$ \\
\hline Sexueller Missbrauch & $25,8 \%$ & $75,2 \%$
\end{tabular}

Auch dieses Jahr sehr ausgeglichene Geschlechterverteilung bei allen Misshandlungsformen ausser beim sexuellen Missbrauch, bei dem Mädchen wiederum dreimal häufiger betroffen waren als Knaben.

Sicherheit der Diagnose

\begin{tabular}{lll} 
Sicher & 902 & $(64,2 \%)$ \\
\hline Wahrscheinlich & 213 & $(15,2 \%)$ \\
\hline Unklar & 288 & $(20,5 \%)$
\end{tabular}

Psychische Misshandlung mit 84,2\% und körperliche Misshandlung mit 65,9\% wurden am ehesten als sicher klassifiziert. Diese Sicherheit war im Falle von Vernachlässigung nur in gut der Hälfte der Fälle, beim sexuellen Missbrauch in knapp der Hälfte der Fälle gegeben.

Täterin/Täter: Beziehung zum Kind

\begin{tabular}{lll} 
Familie & 1097 & $(78,1 \%)$ \\
\hline Bekannte/r des Kindes & 178 & $(12,7 \%)$ \\
\hline Fremdtäter & 36 & $(2,6 \%)$ \\
\hline Unbekannter Täter & 94 & $(6,7 \%)$
\end{tabular}




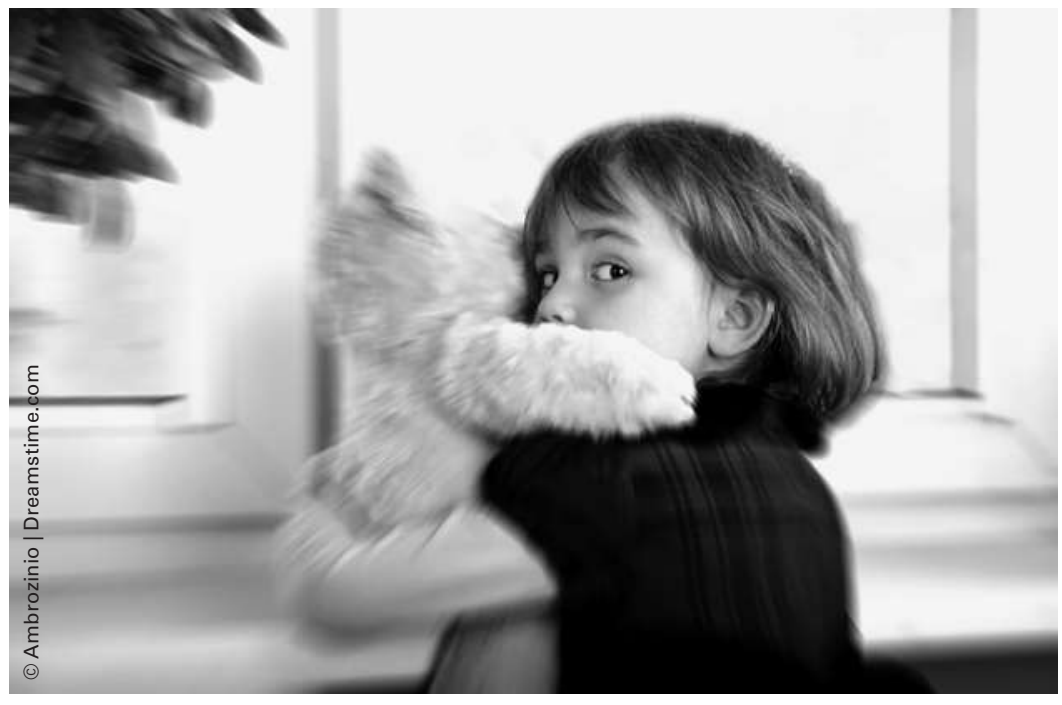

Die Fälle von registrierten Kindsmisshandlunegn haben um weitere $9 \%$ gegenüber dem Vorjahr zugenommen.

Korrespondenz:

Dr. med. Markus Wopmann

Fachgruppe Kinderschutz

Klinik für Kinder

und Jugendliche

Kantonsspital

CH-5404 Baden
Wie auch schon in den letzten Jahren zeigte sich, dass Vernachlässigung oder psychische Misshandlung praktisch immer im Familienrahmen stattfinden. Bei der körperlichen Misshandlung ist dies in 78\% der Fall, beim sexuellen Missbrauch in $42 \%$.

\begin{tabular}{lll}
\hline Täterin/Täter: Geschlecht & & \\
Männlich & 656 & $(46,7 \%)$ \\
\hline Weiblich & 362 & $(25,8 \%)$ \\
\hline $\begin{array}{l}\text { Männlich und weiblich } \\
\text { (meist Eltern gemeinsam) }\end{array}$ & 252 & $(17,9 \%)$ \\
\hline Unbekannt & 135 & $(9,6 \%)$ \\
\hline
\end{tabular}

Sexueller Missbrauch wurde in $87 \%$ der Fälle durch männliche Täter ausgeübt. Im Falle von Vernachlässigung wurde in fast der Hälfte der Fälle eine weibliche Person als Verursacherin bezeichnet, in $40 \%$ aber auch Männer und Frauen (in der Regel die Eltern) gemeinsam.

Täterin/Täter: Alter

\begin{tabular}{lll} 
Älter als 18 Jahre & 1166 & $(83,0 \%)$ \\
\hline Jünger als 18 Jahre & 141 & $(10,0 \%)$ \\
\hline $\begin{array}{l}\text { Jünger und älter als 18 Jahre } \\
\text { (mehrere Täter) }\end{array}$ & 6 & $(0,4 \%)$ \\
\hline \begin{tabular}{l} 
Unbekanntes Alter / keine Angabe \\
\hline
\end{tabular} & 92 & $(6,6 \%)$
\end{tabular}

Beim sexuellen Missbrauch sind 25\% der Täter/Täterinnen jünger als 18 Jahre alt. Erstaunlicherweise beträgt der Anteil der Jugendlichen Täter auch bei der körperlichen Misshandlung 11,4\%.

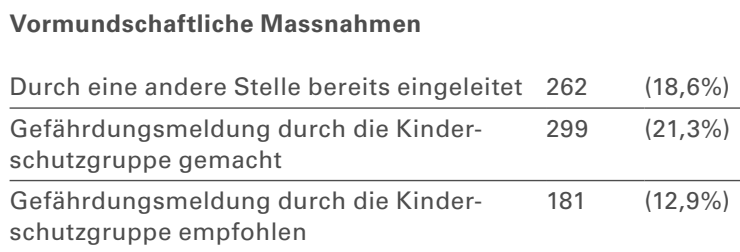

Strafrechtliche Massnahmen

\begin{tabular}{lrl} 
Durch andere Stellen bereits eingeleitet & 184 & $(13,1 \%)$ \\
\hline Durch die Kinderschutzgruppe veranlasst & 97 & $(6,9 \%)$ \\
\hline Durch die Kinderschutzgruppe empfohlen & 75 & $(5,3 \%)$
\end{tabular}

\section{Fazit}

- An schweizerischen Kinderkliniken wurden 2014 mit 1405 Fällen so viele Kinder wie nie zuvor wegen Kindsmisshandlung registriert.

- Die Fälle von psychischer Misshandlung nehmen zu, da bei den gesamtschweizerisch vielen Fällen von häuslicher Gewalt sehr oft Kinder zumindest indirekt mitbetroffen sind.

- In jedem 5. Fall entschloss sich die Kinderschutzgruppe zu einer Gefährdungsmeldung an die Kindes- und Erwachsenenschutzbehörde, in 7\% der Fälle wurde durch die Kinderschutzgruppe eine Strafanzeige veranlasst. In vielen Fällen waren die entsprechenden Behörden bereits vorher in diesen Familien involviert, was zum Ausdruck bringt, dass Kindsmisshandlung häufig in sozial schwierigen und belasteten Situationen vorkommt.

- Tragischerweise sind allein an schweizerischen Kinderkliniken im Jahre 2014 erneut drei Kinder an Kindsmisshandlung verstorben. Zwei Kinder starben an körperlicher Misshandlung und waren jünger als ein Jahr alt, ein Kind starb an Vernachlässigung und war zwischen 1 und 2 Jahre alt. Dies unterstreicht - zusammen mit der Häufigkeit von Kindsmisshandlung bei kleinen Kindern - die hohe Verletzlichkeit und damit auch die hohe Schutzbedürftigkeit in dieser Altersklasse. 